\title{
Characterization of Rational Numbers Using Kronecker's Orbit: A Didactic Approach
}

\author{
Petros-Stylianos Marsellos \\ Greek Ministry of Education, Athens, Greece \\ Email: petros.marsellos@gmail.com
}

How to cite this paper: Marsellos, P.-S. (2018). Characterization of Rational Numbers Using Kronecker's Orbit: A Didactic Approach. Creative Education, 9, 2546-2560. https://doi.org/10.4236/ce.2018.915193

Received: October 8, 2018

Accepted: November 18, 2018

Published: November 21, 2018

Copyright (C) 2018 by author and Scientific Research Publishing Inc. This work is licensed under the Creative Commons Attribution International License (CC BY 4.0).

http://creativecommons.org/licenses/by/4.0/

\begin{abstract}
For every real number $x$, we define as integer part the biggest integer $k$ so that $k \leq x$ and is expressed $[x]$. The difference of the number from its integral part is defined as decimal part of $x$ and expressed with $(x) \in[0,1)$. Consequently, for every $x$, the Kronecker's orbit is defined, namely the set $\{(n x) \mid n \in \mathbb{Z}\}$. Through Kronecker's orbit, rational numbers are characterized as the numbers whose orbit is a bounded set, while irrational numbers are characterized as the numbers whose orbit is a dense set. Using this fundamental theoretical result and utilizing a computer, a didactic approach was established, initially referring to the definition of rational numbers as fraction equivalence classes and basically to the segregation of rational and irrational numbers. This didactic approach also incorporates elements of ancient Greek mathematics history. The proposition was applied to students and was evaluated.
\end{abstract}

\section{Keywords}

Kronecker's Orbit, Rational Numbers' Characterization, Mathematical Education, Geogebra, Decimal Part

\section{Introduction}

In 1881, Leopold Kronecker defined what is called the "rationality domain", which is indeed a body of polynomials in modern terms. In 1893, Heinrich M. Weber gave the first clear definition of the abstract body. In 1910, Ernst Steinitz published a very important article on the Algebraische Theorie der Korper. In this article, he studied the properties of the domains and defined very important theoretical concepts, such as the prime field, perfect field and the field's degree of transcendence. Emil Artin developed the relationship between the groups and the fields with great precision from 1928 to 1942 (Cohen, 2013). 
With regard to Kronecker, whose theory is the basic idea and the basic content of this article, he begins his study with the word "Jugendtraum" as his guide, which is the German translation of the phrase "youthful dream". Indeed, in his letter to Dedekind, Kronecker described as his youthful dream the finding of functions whose specific values could produce abelian extensions of random algebraic fields of numbers. More specifically, he said: "It is the most coveted dream of my youth, namely to prove that abelian equations with explicit square roots date back to transformations of elliptic functions with particular moduli, just as in the case of reduction of integer abelian equations in cyclotomic equations" (Vlăduț, 1991).

The Jugendtraum of Kronecker refers to achieving the construction of all abelian extensions of a squared complex algebraic field of numbers, attaching specific values of certain transcendental functions on any algebraic fields of numbers (Cassels, 1992). When the chosen field is $Q$, the answer to this problem is the Kronecker and Weber theorem. This informs us that any abelian extension of $\mathrm{Q}$ is contained in a cyclotomic field. In other words, each abelian extension of $\mathrm{Q}$ is contained in a field resulting from attachment to $\mathrm{Q}$ of a specific value of the exponential function.

The Jugendtraum is the generalization of the Kronecker and Weber theorem in the case where the algebraic field chosen is not $\mathrm{Q}$, but a random squared complex number field. The effort to solve this problem has led to the development of mathematical disciplines, such as complex multiplication theory and the class field theory (Charollois \& Sczech, 2016).

From the above, it becomes clear that the study of the Jugendtraum requires knowledge and manipulation of sophisticated mathematical concepts and generally theories. For this reason, the choice of analysis of concepts and the presentation of theory to students is not only aimed at achieving the goal of solving the Jugendtraum in the case of squared number fields but also in acquaintance with several attractive mathematics. This article is a reference to the characterization of rational numbers through the use of Kronecker's theorem. The present article has a teaching aim and trend and through the sections that will follow this will not be the only to be explained, but also a thorough analysis of its dynamics at the teaching level will be made.

The sections for the development of this refer to the literature approach of the concept under consideration, and also to the difficulties and constraints identified in rational-irrational numbers, making special reference to technology in education. The article will then refer to the teaching material and generally to the teaching process that should be developed in mathematical education for the better understanding of the present theoretical area, the applications and generally the data that characterize it. The article continues by linking of technology and mathematics to the teaching process. Finally, the article will show the use of Kronecker's chosen model and related scenarios will be presented. Essentially, at the conclusion of the study, not only the theorem and the philosophy of Kro- 
necker will be explained, but also a complete picture of the teaching process that should be followed to understand this and its applications in mathematical science will be provided.

\section{Literature Review Regarding the Difficulties in Understanding the Difference between Rational-Irrational Numbers and Usage of Technology in Education}

Students experience obstacles in understanding real numbers and rational numbers in particular (Smith et al., 2005). These problems still remain among students of schools of mathematics (Hart \&Sinkinson, 1988; Fischbein, Jehiam, \& Cohen, 1995) but also among mathematic teachers. Students do not comprehend the concept of rational numbers and believe that they have nothing to do with real numbers (Moseley, 2005).

- The problem of understanding rational numbers

Rational numbers, as a creation of human thought is a difficult notion (Pitkethly \& Hunting, 1996). Students, having created an impression on numbers (being taught the natural numbers), find it hard to understand rational numbers (Ni \& Zhou, 2005).

Rational and natural numbers present similarities which are only superficial. As a result, students believe that natural numbers' properties are also applied on rational numbers (Kollias et al., 2004; Voskoglou \& Kosyvas, 2012). This student difficulty is reinforced by the fact that they come into contact for the first time with new mathematical symbolisms, which they must understand but also learn to use (Ni \& Zhou, 2005).

Fractions trouble particularly students, who cannot understand that a fraction is a mathematical entity and not two different numbers, they are being taught fractions as part of the whole, and therefore, in the course of their mathematical knowledge, cannot comprehend their further meanings.

Multiple representations of real numbers have a negative effect on their comprehension and students fail to understand that different representations may represent the same number. However, school books do not emphasize on this matter (Voskoglou \& Kosyvas, 2012).

Studies conducted among school and university students (aged 18 - 19 years old, i.e. university students whose mathematical knowledge is limited to what was acquired at school) showed that many of them believe that a number can be rational and irrational, a number may be neither rational nor irrational, a number may not have a decimal representation, a number is real if it has a finite number of decimal digits (otherwise it is not a real number) and that there are real numbers which do not have a decimal representation (Giannakoulias, Souyoul, \& Zachariades, 2007). Students who have not understood rational numbers cannot identify irrational ones, as they believe that fractions are rational while roots are irrational numbers (Voskoglou \& Kosyvas, 2012). These studies showed a general weakness in multiple representations and in the recogni- 
tion of real, rational and irrational numbers (Giannakoulias, Souyoul, \& Zachariades, 2007; Voskoglou \& Kosyvas, 2012). Moreover, it was ascertained that age, along with the depth of mathematical knowledge affect comprehension of these difficult concepts (Giannakoulias, Souyoul, \& Zachariades, 2007).

- How to tackle the problem

Several opinions have been expressed about the ways through which this difficult matter of number comprehension, and particularly rational numbers, can be dealt with. These opinions are based on observed problems but also on the way in which the human mind faces these mathematical concepts. In order to introduce students to these new notions, a radical change of the "number concept" is required (Carpenter et al., 1999). This can be achieved through the contribution of the professor, who, among others, must take into consideration that they are addressing children and not adults, and must therefore follow a corresponding teaching and pedagogical approach. Professors must have full knowledge of real numbers and their subsets, and be equipped with perseverance and patience, emphasizing on solving the students' problems. Also, positive impact could be observed when approaching these concepts with "tangible", representing examples, which are connected with real life.

According to Keiren (1976), when rational numbers are represented on the numbers' line, then it is easier to understand that rational numbers are a subcategory of the real numbers. Furthermore, students are in a position to understand more easily rational numbers, if they learn and become familiar with multiple representations (Kieren, 1980, 1993). For this reason, the teacher can focus on multiple representations using many examples and exercises, exercises which will be done during class by students, working in groups, but also exercises which will be given to students to be solved, so that they also practice at individual level, realising themselves their difficulties and questions. Professors must encourage open discussion to track students' problems and adjust their teaching according to the needs of their class concerned (Voskoglou, 2009).

Finally, we should refer to the opinions according to which rational numbers must be taught at a younger age, even before students form an idea on what a number actually is. When an opinion has been completed, it is very difficult to change the attempt in the future (Voskoglou \& Kosyvas, 2012).

"Question: Why did you answer that $-\frac{\sqrt{5}}{2}$ is a rational number?

Answer: Because it is a fraction.

In this case we have a classical misinterpretation of the definition of rational numbers. The student focused her attention on the symbol of fraction without realizing that, in order to be rational number, its terms must be integers, with non-zero denominators.

Question: Why did you answer that $-\sqrt{4}$ is an irrational number?

Answer: Because it has the root.

Here the student identifies the symbol of the root with an irrational number. He does not think that the given number is equal to -2 , which is an integer. Dis- 
tinction among several types of numbers remains muddy in general, each time depending on their semiotic representations" (Voskoglou \& Kosyvas, 2012).

This represents a very usual student opinion: fractions are rational numbers and roots are irrational numbers.

\section{- Conclusions}

Many researches and studies on students' difficulty in understanding rational numbers have been carried out. Incomplete knowledge of real numbers and their subsets was ascertained from those studies. Also, weakness is observed in recognizing rational-irrational numbers and in using multiple representations. School books are responsible for this, too, as they do not delve into these concepts and therefore, the professor's contribution is crucial for dealing with these difficulties, by him insisting on these problems and "listening" to their students, promoting discussion in class.

- The proof

Teaching proofs is a difficult part of mathematics. Students cannot comprehend proofs and believe that there is no substantial reason for their obligatory teaching.

- The contribution of technology

Technology can help students change their attitude regarding these problems, particularly by re-defining their opinions on geometry and geometrical proof. Since 1980, software of Dynamic Geometry, known as DGS, has been created, aiming to understand Geometry and geometry proofs. These programs may help students comprehend the above and make them realize the importance of proofs, and also be used as an alternative proposition to their approach.

DGS are programs of geometrical visualisation (Cabri, Sketchpad, EukliDraw, a.o.). Through this software, students become active members of knowledge, understand geometry better, are leaded to conclusions, express questions and conjectures.

Studies have shown that DGS can help students understand the need for proofs and intervene to their transition from the intuitive to the theoretical level (Jones, 2000). Of course, there are critics against these programs, who believe that there is a possibility that the teacher's role is lost due to DGS.

There are many students who present difficulties in using rulers and compasses, and DGS solve this problem, as the user can easily construct geometric shapes and confirm their correct or wrong construction.

Basic characteristics of DGS are geometric shapes, geometric relations which characterise geometric shapes and dragging as one of the DGS' most important properties (Cabri, Sketchpad). Through dragging, the user, when creating a construction, can move certain elements of the shape and observe how the shape responds to these changes. It is an observable action, which can lead students to express conjectures and also revise them. Moving and reshaping shapes by preserving the basic relations and properties is possible in DGS (Laborde et al., 2006). 
Students create, change and observe, cease being passive knowledge receivers, become active members and knowledge producers. Calculation and dragging possibilities provide analyses and also properties' discoveries. Students can formulate and revise conjectures through DGS, based on image and numeric data changes; they can revise relations but also work on constructions which copy real-life problems (Kordaki \& Mastrogiannis, 2006).

- Conclusions

DGS use when teaching Geometry and geometric proofs may contribute positively in changing the students' attitude across those matters and also help them express conjectures. The professors who will include DGS in their lesson can create a research laboratory environment, integrating students in groups, acting as their partner, exciting in this way their students' interest. They will guide the students and will provide the scientific verification of their results.

\section{The Role of Technology in Teaching and Understanding Mathematical Concepts}

There are numerous advantages in using technological means both for students and for teachers.

Students:

- Gain access in various sources, information, knowledge from all over the world.

- Are more easily led to create conjectures and they participate actively in the lesson's course.

- Can evaluate themselves.

- Do not feel that they are being judged because the computer is impersonal, and therefore they take initiatives and control their knowledge.

- Can seek distance help.

Teachers:

- Can present images and material with great accuracy in shapes or simulate experiments.

- Can easily have access in a variety of pre-written exercises, e.g.

http://digitalschool.minedu.gov.gr/.

Finally, the computer environment is more attractive and easy-to-use for both groups than the traditional paper and pencil.

However, there are also disadvantages. The most important is that students tend to consider the computer's answer as proof. Also, their high acquisition cost is the main cause for the lack of equipment and software in schools. Lastly, the intensification of the alienation due to lack of human contact is also a disadvantage.

Students usually believe that when a computer presents a geometrical space in a geometrical problem or gives a numeric solution to an algebraic problem, this is automatically proven. This must be taken into consideration and we have to explain that the computer is used to help us make a conjecture, but proof is achieved only when carried out based on mathematical principles and proce- 
dures. The creation of conjectures and the development of intuition are useful tools when trying to find the solution to a problem. Often, if students get the answer-solution through the computer, they can also more easily find the strict proof. The phrase of Professor P. Spyrou in his class is characteristic: "the visualization of the solution (shape) is like the plane's auxiliary wheels; while they are not doing anything during flight, they are necessary for take-off”. There comes the moment when the teacher must point out that mathematics are based on deductive inference and are built only by strict proofs, because intuition can be misled many times.

\section{Characterization of Rational-Irrational Numbers Using Kronecker's Orbit}

Definition: Consider $x \in \mathbb{R}$. The set $O_{x}=\{(n x) \mid n \in \mathbb{Z}\}$ where $(x)=x-[x]$ we symbolize the number's fraction part.

\section{Proposition 1}

Consider $x \in \mathbb{R}$. Then $x \in \mathbb{Q} \Leftrightarrow O_{x}$ is a finite subset of $[0,1)$

\section{Proof}

Consider $x=\frac{k}{l}, k \in \mathbb{Z}, l \in \mathbb{N},(k, l)=1$. Then $(k x)=(0)=0$, therefore $O_{x}=\{0,(x), \ldots(k x)\}$ is finite

Vice versa, if $O_{x}$ is finite, then $\exists m, n \in \mathbb{Z}, m<n:(m x)=(n x)$. So,

$$
n x-m x=k \in \mathbb{Z} \text { therefore } x=\frac{k}{n-m} \in \mathbb{Q}
$$

\section{Proposition 2}

Consider $x \in \mathbb{R}$. Then $x$ irrational $\Leftrightarrow O_{x}$ is a dense subset of $[0,1)$

Proof

If $O_{x}$ is a dense subset of $[0,1)$ then it is infinite, so from the previous proposition $x$ irrational.

$>$ Vice versa, consider irrational. I will show that for any y of $[0,1)$ there is an element of $O_{x}$ which is close to y. That is, for

$y \in[0,1), 0<e<\frac{1-y}{2} \exists v \in \mathbb{Z}$ with $(n x) \in O_{x}$ so that $|(n x)-y|<e$. I choose $\mathrm{M}=\left[\frac{1}{e}\right]+1 \in \mathbb{N}$. I divide $[0,1)$ in $\mathrm{M}$ equal intervals and I take the first $\mathrm{M}+1$ elements of $O_{x}$ from 0 , namely $0,(x), \ldots,(\mathrm{M} x)$. Using the pigeonhole principle since I have $M+1$ elements and $M$ intervals, this means that at least two elements $(\mathrm{kx})$, (lx) with $0<\mathrm{k}<1<\mathrm{M}$ belong to the same interval and therefore their distance is less than $\frac{1}{\mathrm{M}}:|(k x)-(l x)|<\frac{1}{\mathrm{M}}$. Since $\mathrm{x}$ is irrational, then $(k x) \neq(l x)$ and I put $0<r=l-k \leq l \leq \mathrm{M}$ and $j=[l x]-[k x]$. We have $|r x-j|=|(k x)-(l x)|<\frac{1}{\mathrm{M}}<e, j \in \mathbb{Z}$. So, $[r x]=j$ or $[-r x]=-j$ and $(r x)<e$ or $(-r x)<e$. So, there is an $\in \mathbb{N}:(s t x) \leq y<((s+1) t x)$. Therefore, $|(s t x)-y|<e$. 


\section{Scenario Presentation}

Our goal is to create an alternative scenario which will offer a new perspective in the topic of rational numbers using the computer, based on the history and the problems we mentioned above in the literature review. The scenario consists of four papers which are given to the students consecutively, ages: 14 - 16 .

\section{Activity Analysis}

\section{Part one: reminders-additions}

Prerequisites:

Firstly, we will discuss about the fraction part. Here we will remember the Euclidean division and we will define the integer and decimal or fraction part relating it with mixed numbers which students learnt in primary school. Using specific examples, students practice alone in finding numbers' fraction parts. In this activity each one will work in their notebook for about 15 minutes.

After that, a Discussion about representing numbers in the line will be done. They have been already taught this topic, but we will give them some examples to make sure that there will be no mistakes due to this in the questions of page 2 . Completion will be done in pairs under the teacher's supervision. We do not expect students to face particular difficulties.

Afterwards, the students will complete the first page (Table 1). The first column has the numbers " $a$ ", in the second one " $2 a$ " we write number a's double and in the third one " $(2 a)$ " we write $2 a$ 's fraction part as already completed as an example.

\section{Completing Table 1}

\section{Part two: The orbit's concept}

Having completed the table, we ask students to circle fraction parts on the page. "Is there any pattern they are following? How do you believe it would continue if we wrote more multiples?" Here we expect them to find out that fraction parts are repeated. There we will give the name "orbit". We will remind them, if necessary, that in a set we only write once each element. "Which is the orbit for every number of the table?" This question leads us to the second page (Figure 1). Here the orbit is represented in the rectilinear part after being defined as a set.

Table 1. First page of the scenario. Finding the fraction part.

\begin{tabular}{|c|c|c|c|c|c|c|c|c|c|c|c|c|c|c|c|c|}
\hline $\mathrm{a}$ & $2 \mathrm{a}$ & (2a) & $3 a$ & $(3 \alpha)$ & $4 a$ & (4a) & $5 a$ & (5a) & $6 a$ & (6a) & $7 \mathrm{a}$ & (7a) & $8 a$ & (8a) & (9a) & (10a) \\
\hline $1 / 2$ & $2 / 2$ & 0 & $3 / 2$ & & & & & & & & & & & & & \\
\hline $1 / 3$ & $2 / 3$ & & & & & & & & & & & & & & & \\
\hline $3 / 4$ & & & & & & & & & & & & & & 0 & & \\
\hline $1 / 5$ & & & & & & $4 / 5$ & & & & & & & & & & \\
\hline $2 / 3$ & & & & & & & & & & & & & & & & \\
\hline 2 & & & & & & & & & & & 14 & & & & & \\
\hline $3 / 6$ & & & & & & & & & & & & & & & & \\
\hline
\end{tabular}




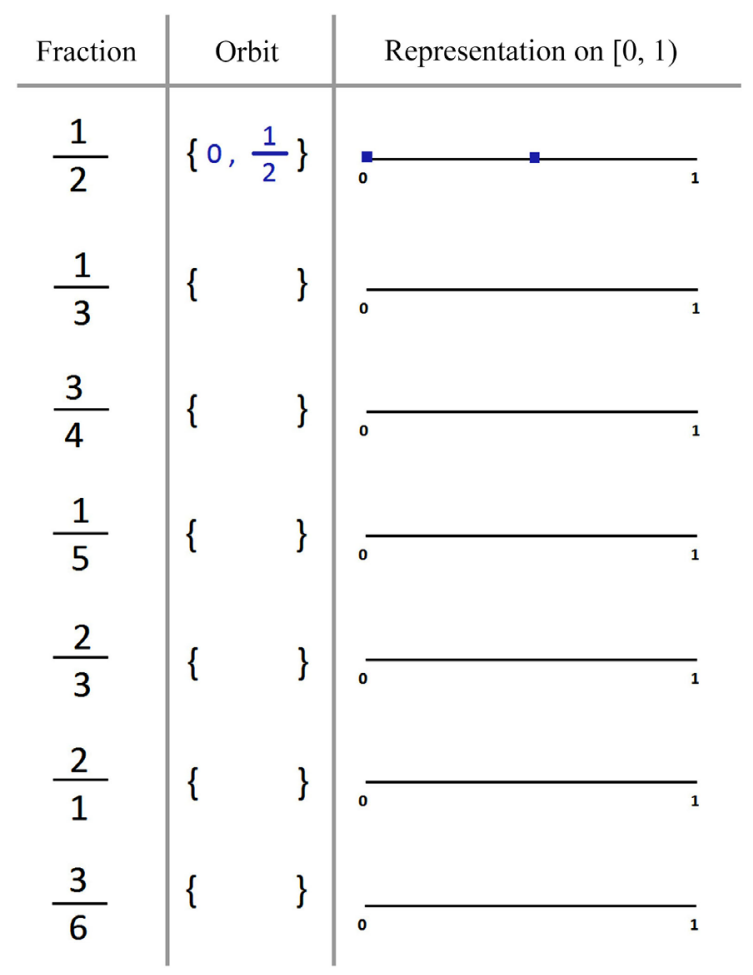

Figure 1. Second page of the scenario. Finding Kronecker's orbit.

We start by completing the orbit's elements, i.e. the second column. The first one is already completed as an example. Once again, students work in pairs. From here we begin our steps to characterize rational numbers. I ask "Of how many elements does the set consist? Write the number of elements next to each set. What do you observe?" Here I want them to correlate the quantity with the denominator. If they do not realise that, I act as follows: I ask "tell me the fraction of the first column" and immediately afterwards "how many elements does the orbit have" and I repeat that in all elements except for the last one. There, the connection is made as soon as they listen to the numbers. It is a bit guiding but it may be necessary. "So, where are we led? To what is this quantity related for each number? Make a conjecture". Here, I expect them to tell me that the quantity of the orbit is equal to the denominator. Then, I bring the last orbit of $3 / 6$ and I ask them if it also applies here. Can you find the particularity of this fraction compared to the other ones? That is, to make them see it is not a fraction in lowest terms. "Transform it into a fraction in lowest terms. Now, does the previous conjecture apply?" Then, I ask them to change appropriately their conjecture in order to include all cases. We discuss for a while the fractions' equivalence. It is obvious that equivalent fraction will have the same orbit. The reverse does not apply. I want them to realize that and that's why I have put $1 / 3$ and $2 / 3$ which have the same sets as orbits. We conclude to the final conjecture: "If we have a fraction in lowest terms $\mathrm{k} / \mathrm{l}$, then the orbit consists of 1 elements". I ask them to write it down. "Your conjecture is correct and thus the implication is double but to make it a proposition we need proof. This can be done later so that 
we do not spoil the lesson's course now. For the time being, accept it because I say to you that I have proved it". The proof is not difficult but in my opinion requires a familiarisation with the concepts we've just learnt and could cause agitation if mentioned altogether. Mentioning that there is a proof is necessary.

Afterwards, the graphical representation of the table's elements follows, in the page's third column. There, the $[0,1)$ can be done with separation in as many equal parts as many elements the set has, or with the traditional representation.

We proceed to Page 3 (Figure 2) which is matching. The definition of rational numbers is: "the numbers which can be written as fractions of integers". This "can" is page 3's main goal.

This is obvious from the choice of forms that are not fractions of integers and the fraction line is next to them. It requests to be completed with the appropriate form which will allow them to apply the proposition they discovered. Of course, through this page I also want to ensure that they can apply correctly the proposition (e.g. in equivalent fractions) and find out the matches without making operations with the fraction parts (Always in pairs).

Part three: the PC

I now explain that through a program we can see orbits for all numbers (I present images) (Figure 3).

"We now turn on the PC. Let's see what you have done ... Firstly, we will confirm some of your results". After we ascertain the correspondence of their results with those of the program, we proceed to the next step.

Now we give them the final page (Figure 4) where the geometrical construction of numbers is presented, and we will check their orbits. Here, we will come to infinite orbits for the numbers we described before as irrational, $-\sqrt{2}$,

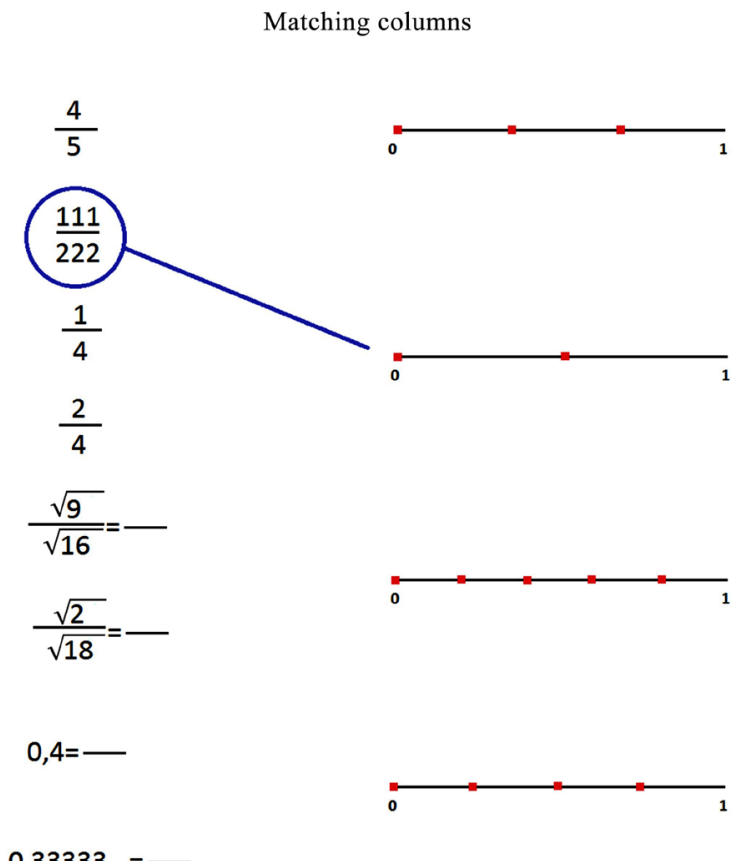

Figure 2. Third page of the scenario. Matching numbers to orbits. 


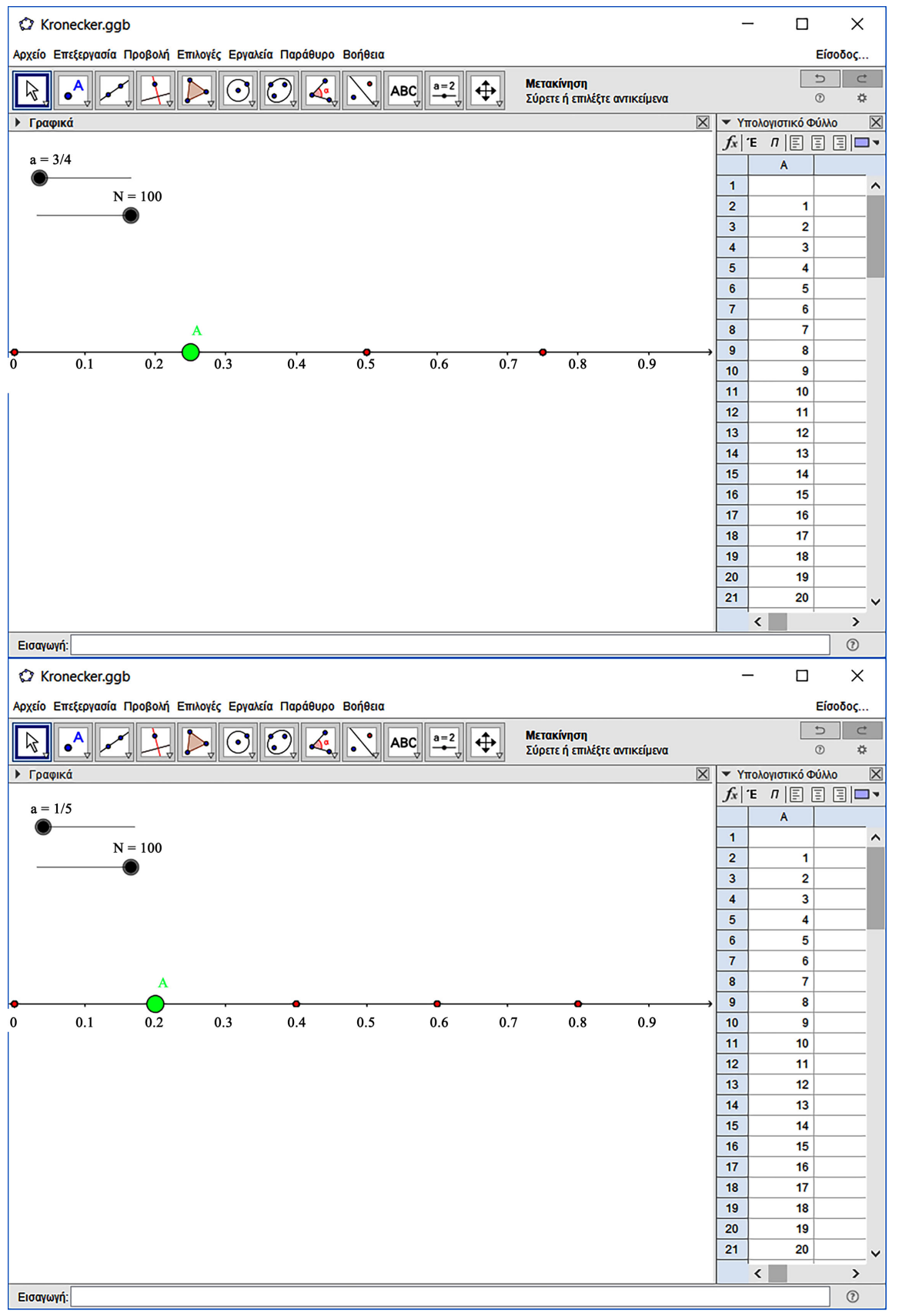

Figure 3. Examples of Kronecker's orbits for rational numbers using Geogebra.

$\frac{1 \pm \sqrt{5}}{2}$, the golden ratio, the ratio of the circumference to the diameter and of the side to the diagonal of a square. "We have learnt in Junior high School that the ratio of the circumference to the diameter gives us?" $\pi=3.14159 \ldots$ "The Pythagorean Theorem states that the ratio of a diagonal to a square's side equals to $1.414 \ldots$... and with a trinomial like that of the school book of the third grade of Junior High School we know that the golden ratio is the ratio $1.618 \ldots$

"If we wanted to see those numbers' orbits, it would be impossible with paper and pencil. Here comes to help us the program we saw before to represent in [0, 
1) in the orbit of those numbers. Let's see".

I indicatively present the image for pi (Figure 5).

I zero the cursor and start raising multiples up to 200 which are more than enough in all three cases to complete with dense dots $[0,1)$.

Part four. irrational numbers

"How many elements does the orbit have here?" $\infty$ infinite.

"Let's remember the proposition we found before. Find it there where we wrote it to see it". If we have a fraction in its lowest terms $\mathrm{k} / \mathrm{l}$, then the orbit consists of 1 elements and vice versa, if the number's orbit consists of 1 elements then the number is written as a fraction in its lowest terms with denominator 1 . And here lies the big question:

"Could they be written as a fraction and, if yes, with which denominator?"

I expect through conversation with the whole team to conclude finally that no, they couldn't. Students may try to make operations using infinity as if it were a real number. This appears to be another delicate point which must be clarified to students. Finally, create the definition and an extra characterization of rational numbers.

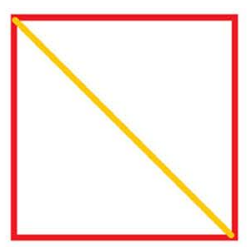

$$
\frac{\text { perimeter }}{\text { diagonal }}=\sqrt{2}=1.414 \ldots
$$
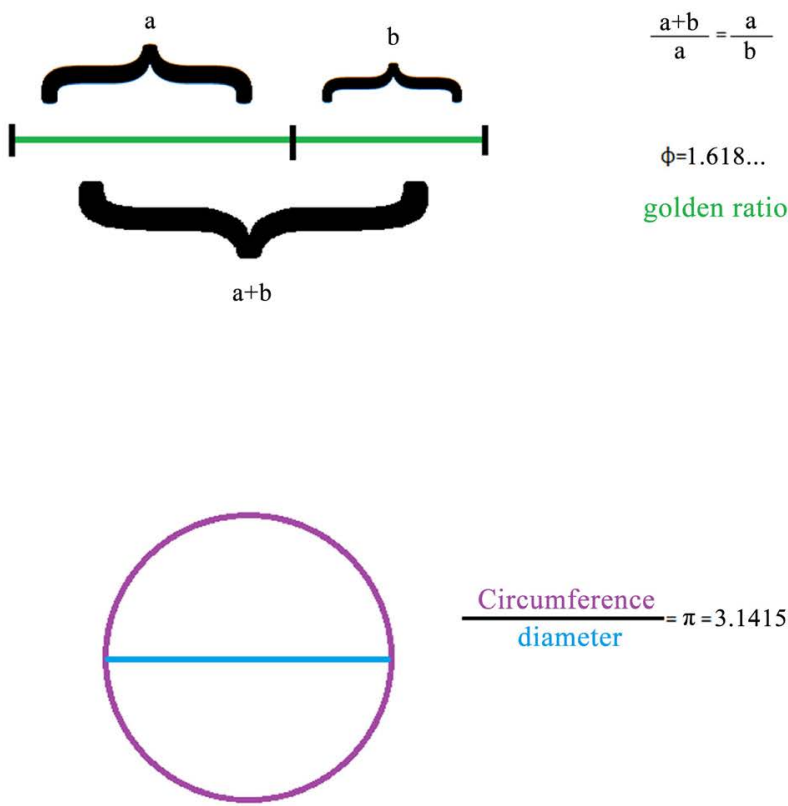

$\frac{\text { Circumference }}{\text { diameter }}=\pi=3.1415926 \ldots$

Figure 4. Final page of the scenario. Introducing assymetry from known geometrical theorems. 


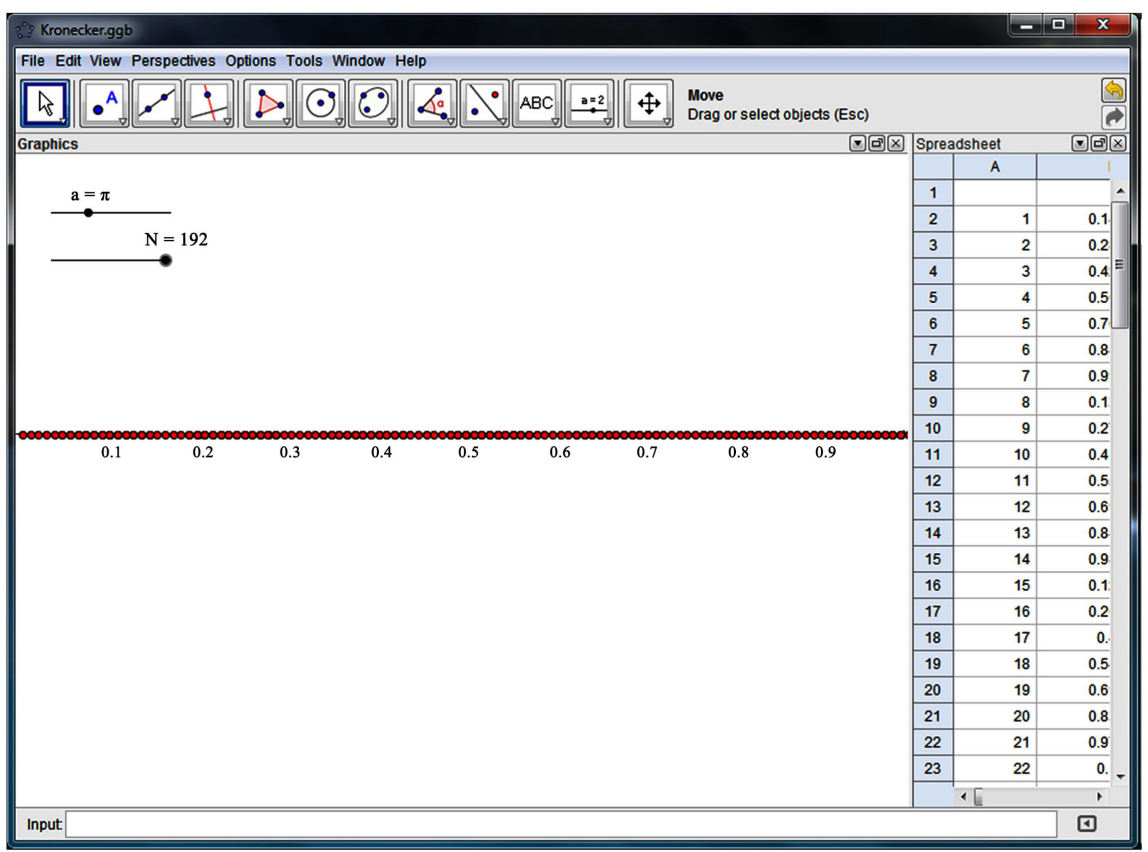

Figure 5. Example of Kronecker's orbit for irrational numbers using Geogebra (Dense).

The typical proof follows.

\section{Definition}

Consider $x \in \mathbb{R}$. The set $0 x=\{(n x) \mid n \in \mathbb{Z}\}$ where $(\mathrm{x})=\mathrm{x}-[\mathrm{x}]$ we represent the number's fraction part, it is called Kronecker's orbit.

\& Proposition (characterization of rational numbers)

Consider $x \in \mathbb{R}$. Then $x \in \mathbb{Q} \Leftrightarrow 0 x$ is an finite subset of $[0,1)$

Proof

Consider $\quad x=\frac{k}{l}, k \in \mathbb{Z}, l \in \mathbb{N},(k, l)=1$. Then $(l x)=(0)=0$, therefore $O_{x}=\{0,(x), \ldots(k x)\}$ is finite.

$>$ In reverse, if $O_{x}$ is finite, then $\exists m, n \in \mathbb{Z}, m<n:(m x)=(n x)$. Therefore, $n x-m x=k \in \mathbb{Z}$ so $x=\frac{k}{n-m} \in \mathbb{Q}$.

\section{Continuation}

It is very important that students understand through ancient problems the concept of asymmetry qualitatively, that is, the non-existence of a common measure and not simply through definitions. In Euclid's Elements, the rational numbers were characterized with a method called anthyferesis and there was proof that the square roots of $2,3 \ldots 17$ were irrational.

\section{Discussion}

Firstly, students get a picture for the concept of irrationality through different representations for the first time. We believe that this will help students in understanding more profoundly rational and irrational numbers. Also, it is very 
important for students to make assumptions, which is the first step when learning a new concept.

\section{Conflicts of Interest}

The author declares no conflicts of interest regarding the publication of this paper.

\section{References}

Carpenter, T., Fennema, E., Fuson, K., Hiebert, J., Human, P., Murray, H. et al. (1999). Learning Basic Number Concepts and Skills as Problem Solving. Mathematics Classrooms that Promote Understanding, 45-61.

Cassels, J. W. S. (1992). Kronecker's Jugendtraum and Modular Functions. Bulletin of the London Mathematical Society, 24, 613-614. https://doi.org/10.1112/blms/24.6.613

Charollois, P., \& Sczech, R. (2016). Elliptic Functions according to Eisenstein and Kronecker: An Update. EMS Newsletter, 9, 8-14. https://doi.org/10.4171/NEWS/101/4

Cohen, H. (2013). A Course in Computational Algebraic Number Theory. New York: Springer Science \& Business Media, Springer-Verlag Berlin Heidelberg GmbH.

Fischbein, E., Jehiam, R., \& Cohen, D. (1995). The Concept of Irrational Numbers in High-School Students and Prospective Teachers. Educational Studies in Mathematics, 29, 29-44. https://doi.org/10.1007/BF01273899

Giannakoulias, E., Souyoul, A., \& Zachariades, T. (2007). Students' Thinking about Fundamental Real Numbers Properties. In Proceedings of the Fifth Congress of the European Society for Research in Mathematics Education (pp. 416-425). Cyprus: ERME, Department of Education, University of Cyprus.

Hart, K., \& Sinkinson, A. (1988). Forging the Link between Practical and Formal Mathematics. In Proceedings of the 12th International Conference for the Psychology of Mathematics Education (Vol. 2, pp. 380-384).

Kieren, T. E. (1980). Five Faces of Mathematical Knowledge Building. Department of Secondary Education, University of Alberta.

Kieren, T. E. (1983). Partitioning, Equivalence and the Construction of Rational Number Ideas. In Proceedings of the Fourth International Congress on Mathematical Education (pp. 506-508). Boston: Birkhauser.

Kollias, B., Mamalougos, N., Mol, A., Vamvakousi, X., Stathopoulou, X., \& Vosniadou, S. (2004). Significant Turns of Teachers' Professional Knowledge who Implement Computer Supported Collaborative Learning Environments. In Proceedings of 4th Conference Technologies of Information and Communication in Education (pp. 45-62).

Kordaki, M., \& Mastrogiannis, A. (2006). The Potential of Multiple-Solution Tasks in E-Learning Environments: Exploiting the Tools of Cabri Geometry II. In E-Learn: World Conference on E-Learning in Corporate, Government, Healthcare, and Higher Education (pp. 97-104). Association for the Advancement of Computing in Education (AACE).

Laborde, C., Kynigos, C., Hollebrands, K., \& Strässer, R. (2006).Teaching and Learning Geometry with Technology. Handbook of Research on the Psychology of Mathematics Education: Past, Present and Future, 275-304.

Moseley, B. (2005). Students' Early Mathematical Representation Knowledge: The Effects of Emphasizing Single or Multiple Perspectives of the Rational Number Domain in Problem Solving. Educational Studies in Mathematics, 60, 37-69. 
https://doi.org/10.1007/s10649-005-5031-2

Ni, Y., \& Zhou, Y. D. (2005). Teaching and Learning Fraction and Rational Numbers: The Origins and Implications of Whole Number Bias. Educational Psychologist, 40, 27-52. https://doi.org/10.1207/s15326985ep4001 3

Pitkethly, A., \& Hunting, R. (1996). A Review of Recent Research in the Area of Initial Fraction Concepts. Educational Studies in Mathematics, 30, 5-38.

https://doi.org/10.1007/BF00163751

Smith, C. L., Solomon, G. E., \& Carey, S. (2005). Never Getting to Zero: Elementary School Students' Understanding of the Infinite Divisibility of Number and Matter. Cognitive Psychology, 51, 101-140. https://doi.org/10.1016/j.cogpsych.2005.03.001

Vlăduț, S. G. (1991). Kronecker's Jugendtraum and Modular Functions 2. Luxembourg: Cordon and Breach Publishers, CRC Press.

Voskoglou, M. G. (2009). Fuzzy Sets in Case-Based Reasoning. In 2009 Sixth International Conference on Fuzzy Systems and Knowledge Discovery (pp. 252-256). https://doi.org/10.1109/FSKD.2009.667

Voskoglou, M., \& Kosyvas, G. D. (2012). Analyzing Students' Difficulties in Understanding Real Numbers. Journal of Research in Mathematics Education, 1, 301-336. 